International Journal of Current Advanced Research

ISSN: O: 2319-6475, ISSN: P: 2319 - 6505, Impact Factor: SJIF: 5.995

Available Online at www.journalijcar.org

Volume 6; Issue 3; March 2017; Page No. 2372-2375

DOI: http://dx.doi.org/10.24327/ijcar.2017.2375.0012

Research Article

\title{
ROLE OF ULTRASOUND GUIDED FNAC IN AXILLARY STAGING IN BREAST CARCINOMA WITH NO CLINICALLY PALPABLE AXILLARY LYMPH NODES. A PROSPECTIVE STUDY
}

\author{
1,2,3 Department of Surgery, IGMC, Shimla(H.P.)171001 \\ ${ }^{4}$ Department of Radiology, IGMC \\ 5Department of Pathology, IGMC
}

V.K Sharma1., Vibhor Nanda ${ }^{2}$., K.J.S. Jaswal ${ }^{3}$., Anupam Jhobta ${ }^{4}$ and Sudershan Sharma ${ }^{5}$

\section{A R T I C L E I N F O}

\section{Article History:}

Received $19^{\text {th }}$ December, 2016

Received in revised form $24^{\text {th }}$ January, 2017

Accepted $12^{\text {th }}$ February, 2017

Published online $28^{\text {th }}$ March, 2017

\section{Key words:}

Axillary lymph node dissection - ultrasound guided FNAC, ALND-axillary lymph node dissection, MRM-modified radical mastectomy, BCS-Breast conservative surgery, USG ultrasonography

\begin{abstract}
A B S T R A C T
The purpose of this study was to stage the axilla by USG guided FNAC in clinically node negative cases and whether cumbersome procedure of sentinel lymph node dissection in breast carcinoma can be avoided for staging of axilla. The present study was carried out in Department of Surgery, IGMC, Shimla in collaboration with the departments of Radiodiagnosis and Pathology over a period of 12 months from 1st July 2015 to 30th June 2016 on 40 patients. Patients of diagnosed breast carcinoma and clinically node negative axilla were included in this study. Ultrasound guided FNAC of suspicious axillary lymph nodes was obtained in every case and sent for histopathology and these findings were compared with those of breast surgery specimen. Majority of the patients in the study were above the age of 50 years (75\%). Ultrasound guided FNAC of suspicious axillary The sensitivity, positive predictive value, and negative predictive value of Axillary USG alone were $85.71 \%, 80 \%$, and $60 \%$ respectively. The overall diagnostic accuracy was $75 \%$. The sensitivity, specificity, positive predictive value and negative predictive value of Axillary USG guided FNAC were $91.66 \%, 100 \%, 100 \%$, and $75 \%$ respectively. The overall diagnostic accuracy of USG guided FNAC was (93\%). We conclude that Ultrasound guided FNAC of non-palpable indeterminate and suspicious axillary lymph nodes is a simple, minimally invasive and reliable technique for the initial determination of axillary lymph node status in breast carcinoma patients and can be immensely valuable in planning the appropriate management of patients, if adopted into routine clinical practice. Patients who have suspicious nodes on ultrasonogram and a subsequently positive FNAC can be considered node-positive and are recommended to undergo Axillary lymph node dissection at the time of primary breast surgery and sentinel lymph node biopsy can be avoided which is much cumbersome and time consuming procedure.
\end{abstract}

Copyright $\odot 2017$ V.K Sharma. This is an open access article distributed under the Creative Commons Attribution License, which permits unrestricted use, distribution, and reproduction in any medium, provided the original work is properly cited.

\section{INTRODUCTION}

Breast cancer is the commonest cancer in women worldwide. The developed countries with a small proportion of the world population account for almost $50 \%$ of breast cancers. In the developing countries of Asia, the health care burden on account of breast cancer has been steadily mounting ${ }^{1}$. Over 100,000 new breast cancer patients are estimated to be diagnosed annually in India Management of breast cancer is a multidisciplinary approach consisting of surgery, chemotherapy, hormone blocking therapy, monoclonal antibodies and radiotherapy. With increased awareness \& screening programs in west, more number of women now present with small tumours, and hence increase in the number of breast conservation surgeries with long term results similar to that of modified radical mastectomy.

*Corresponding author: V.K Sharma

Department of Surgery, IGMC, Shimla(H.P.)171001
Despite the availability of lots of new molecular markers, axillary nodal status still remains the most important prognostic factor for survival in breast cancer. The expected 5 year survival for a node negative breast cancer is over $90 \%$ while the survival drops to below $70 \%$ in those with positive nodes. Axillary lymph node staging is the single most important prognostic indicator for breast cancer and is fundamental prerequisite in the therapeutic decision. For many decades it has been routine practice to surgically remove axillary lymph nodes for histopathological assessment in all women with invasive disease to determine the most appropriate treatment options. However, only $38 \%$ of women with breast cancer have positive nodes ${ }^{2}$.

Current UK guidelines advise that sentinel lymph node biopsy positive women should proceed to completion Axillary Lymph Node Dissection, while node-negative women need no further axillary treatment ${ }^{2}$. While sentinel lymph node biopsy results in considerably less morbidity than Axillary Lymph 
Node Dissection $[\mathrm{ALND}]^{3}$, it does require radioisotope and/or blue dye injection, and usually general anaesthesia. If axillary metastatic disease can be identified prior to surgery, nodepositive patients can avoid sentinel lymph node biopsy and have Axillary Lymph Node Dissection as part of their primary surgery. Physical examination alone is very unreliable and has an accuracy of only 30-60\% when compared with $\mathrm{ALND}^{4}$. Axillary dissection is associated with morbidities including shoulder and arm pain, lymphoedema of arm, seroma and lymphosarcoma. Axillary ultrasound is the primary nonsurgical method for evaluating axillary nodes. It is moderately sensitive and can be highly specific, especially when morphologic criteria are used as the primary diagnostic criteria, With the addition of USG-guided fine-needle aspiration or core needle biopsy of suspicious lymph nodes, sensitivity and positive predictive value can be increased ${ }^{8,9,10}$. The employment of axillary ultrasound and fine needle aspiration cytology of axillary nodes in the preoperative period, allows the surgeon to avoid or perform axillary dissection in node negative or positive patients respectively as a single step surgery. We conducted this study to find out the diagnostic strength of Ultrasound and ultrasound guided FNAC of suspicious axillary lymph nodes preoperatively during the routine work up and to plan our treatment accordingly without compromising oncological principles.

\section{METHODS \& MATERIALS}

This study was conducted on 40 patients of proven carcinoma breast over a period of 1 year (from July 2015 to June 2016). Patients who were clinically N0 were selected to undergo Axillary Ultrasound. This study was approved by the ethical committee of our institution. Written consent was obtained from the patients included in this study. All patients with cytologically proven Breast Carcinoma with no axillary lymph nodes palpable clinically regardless of age, comorbidity, size of lump, stage of carcinoma were included while patients who underwent Axillary lymph node dissection previously, patients with clinically palpable axillary lymph nodes and patients who refused to be part of the study were excluded from the study. All patients who were found to have suspicious Lymph nodes on Axillary Ultrasound were chosen to undergo Ultrasound Guided FNAC of the suspicious nodes which was done by the same radiologist in every case. The suspicious node on ultrasound was defined according to guidelines of Baruah et al. ${ }^{11}$ The features of a suspicious node taken on ultrasound were: 1. Longitudinal to transverse axis of node ratio of less than 2. 2. Focal eccentric cortical thickening of more than $2 \mathrm{~mm}$. 3.Concentric cortical thickening of more than $3 \mathrm{~mm}$. 4. Compression or obliteration of nodal fatty hilum. 5. Ill-defined nodal margins.

Patient was placed supine and axilla of the side of pathology was examined with a $12 \mathrm{MHz}$ ultrasound probe. On detecting any suspicious nodes, USG guided FNAC was taken with a $22 \mathrm{G}$ needle attached to a $20 \mathrm{cc}$ syringe.

Smears were prepared from the aspirate, air dried and sent for cytological evaluation. All patients were subjected to surgery (MRM/BCS) along with axillary dissection and the histopathological report of the axillary nodes was taken as reference and compared with previous USG guided FNAC report.

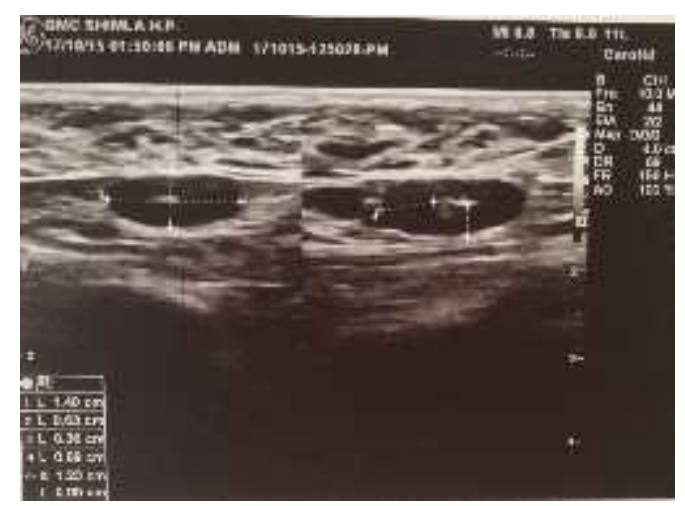

USG axilla with LAP, Longitudinal and transverse axis measurement in left, cortical thickness measurement in right.

\section{RESULTS}

Out of 40 patients, $30(75 \%)$ had suspicious nodes on axillary USG whereas $10(25 \%)$ were having benign nodes. These 30 patients having suspicious nodes were subjected to FNAC of nodes from the suspicious areas and out of these 30 cases 22 $(73.33 \%)$ were found to have nodal metastasis on cytological evaluation and $8(26.67 \%)$ were found be negative. On HPE of surgery specimens of 40 patients, $28(70 \%)$ were found to be positive for nodal metastasis whereas 12(30\%) patients were negative for nodal metastasis. Histopathology of surgery specimen was taken as the gold standard for detection of axillary node metastasis. 24 out of 30 patients having suspicious lymph nodes on USG axilla had nodal metastasis positive on histopathological examination as well whereas the other 6 were negative for nodal metastasis on histopathological examination. 6 out of 10 patients who had benign nodes on ultrasound axilla had negative nodal metastasis whereas 4 had metastasis positive on histopathological examination as depicted in table-1.

Table1 Showing Concordance between USG axilla \& HPE

\begin{tabular}{ccc}
\hline USG axilla & HPE & $\begin{array}{c}\text { No. of } \\
\text { patients }\end{array}$ \\
\hline Positive & Positive & 24 \\
Positive & Negative & 6 \\
Negative & Positive & 4 \\
Negative & Negative & 6 \\
\hline
\end{tabular}

All 22 patients who had nodal metastasis positive on USG guided FNAC had nodal metastasis positive on histopathological examination as well whereas 2 out of 8 patients having no metastasis on FNAC had nodal metastasis positive on histopathological examination as shown in table- 2 .

Table2 Showing Concordance between USG guided FNAC \& HPE

\begin{tabular}{ccc}
\hline USG guided FNAC & HPE & No. of patients \\
\hline Positive & Positive & 22 \\
Positive & Negative & 0 \\
Negative & Positive & 2 \\
Negative & Negative & 6 \\
\hline
\end{tabular}

Using statistical equations following results were observedThe sensitivity, positive predictive value (PPV), and negative predictive value (NPV) of Axillary USG alone were $85.71 \%$, $80 \%$, and $60 \%$ respectively. The overall diagnostic accuracy was $75 \%$. The sensitivity, specificity, positive predictive value and negative predictive value (NPV) of Axillary USG 
guided FNAC in our study were $91.66 \%, 100 \%, 100 \%$, and $75 \%$ respectively. The overall diagnostic accuracy of US guided FNAC was $93.33 \%$.

\section{DISCUSSION}

In this study we found that the sensitivity, positive predictive value (PPV), and negative predictive value (NPV) of Axillary USG alone were $85.71 \%, 80 \%$, and $60 \%$ respectively. The overall diagnostic accuracy was $75 \%$. Fernandez et $a l^{9}$ found that when compared with final axillary histology, ultrasound fine-needle aspiration showed positive predictive value of $87 \%$, negative predictive value of $82 \%$, sensitivity of $53 \%$ and specificity of $100 \%$. Leenders et $a l^{12}$ reported sensitivity, specificity, positive predictive value (PPV) and negative predictive value (NPV) of axillary ultrasound alone as $43.8 \%$, $80.7 \%, 57.5 \%$ and $70.7 \%$ respectively. Feng Y, et al ${ }^{13}$ reported sensitivity, specificity, PPV, NPV, and accuracy of axillary ultrasound alone as $58.6 \%, 89.4 \%, 79.6 \%, 75.3 \%$, and $76.7 \%$, respectively. A Moore et $a l^{14}$ reported in their study that the overall sensitivity and specificity values for sonography alone were $81 \%$ and $69 \%$ respectively. Due to overlapping sonographic features of benign/reactive and suspicious/metastatic lymph nodes, a large number of lymph nodes that would otherwise be categorized as indeterminate for metastasis can be more definitively diagnosed if ultrasound is combined with FNAC. We found that the sensitivity, specificity, positive predictive value and negative predictive value (NPV) of Axillary USG guided FNAC in our study were $91.66 \%, 100 \%, 100 \%$, and $75 \%$ respectively. The overall diagnostic accuracy of USG guided FNAC was 93.33\%. Leenders et $a l^{12}$ reported sensitivity, specificity, PPV, NPV of Axillary USG - FNAC as 24.7\%, 99.9\%, $99.1 \%$ and $69.0 \%$ respectively. \%. Feng $\mathrm{Y}$, et $a l^{13}$ reported sensitivity, specificity, PPV, NPV, and accuracy of FNACUSG combine of $52.4 \%, 100 \%, 100 \%, 74.8 \%$, and $80.3 \%$, respectively. Bonnema et $a l^{15}$ who performed US-guided FNA of 122 lymph nodes obtained from 81 axilla and found a sensitivity of $80 \%$ and a specificity of $100 \%$. Carroll et al ${ }^{16}$ in their study evaluated the combined role of axillary ultrasound and fine needle aspiration and concluded that Axillary USGFNAC had a sensitivity of $63.4 \%$ and specificity of $100 \%$. Krishnamurthy et $a l^{17}$ found that USG-FNAC had specificity of $84.6 \%$ and sensitivity $84.2 \%$, the diagnostic accuracy was $79 \%$, the PPV was $100 \%$, and the NPV was $67 \%$. Verbanck et $a l^{18}$ reported the sensitivity of USG-FNAC in detecting malignant nodes was $92 \%$, the specificity $95 \%$, and the PPV and NPV $96 \%$ and $91 \%$, respectively. Holwitt DM, et al ${ }^{19}$ reported sensitivity and specificity of USG-FNAC as $71 \%$ and $99 \%$, with a NPV of $84 \%$ and PPV of $97 \%$.

We had $2 / 30(6.66 \%)$ false negative results. Bonnema et $a l^{15}$ reported a false negative result of $12 \%$. Krishnamurthy et al reported ${ }^{17} 12 / 103(11.6 \%)$ as false negative cases. Holwitt et al ${ }^{[19]}$ reported false negative rate of $8 \%$ (9 out of 110 patients). False-negative FNAC results probably occurred in part due to failure to target the real SLN or the most suspected region of the lymph node and perhaps misinterpretations including failure to recognize tumour cells.

\section{CONCLUSION}

Ultrasound guided FNAC of non-palpable indeterminate and suspicious axillary lymph nodes is a simple, minimally invasive and reliable technique for the initial determination of axillary lymph node status in breast carcinoma patients. The high sensitivity, specificity and PPV and relatively low falsenegative rate of USG- guided FNA of non-palpable axillary lymph nodes indicate that it is a useful procedure in the initial staging of breast carcinoma and can be immensely valuable in planning the appropriate management of patients if adopted into routine clinical practice. Patients who have suspicious nodes on ultrasonogram and a subsequently positive FNAC can be considered node-positive and are recommended to undergo Axillary Lymph Node Dissection at the time of primary breast surgery itself. In this group of patients sentinel lymph node biopsy can be avoided which is much cumbersome and time consuming procedure. However a study with a much larger population may help to further substantiate the findings observed in the present study.

\section{References}

1. Mir R, Singh V et al. Breast cancer in young women and its impact on reproductive function. Apollo Medicine, September 2009:Vol. 6(3):200-208.

2. NHS Cancer Screening Programmes. All Breast Cancer Report. A UK analysis of all symptomatic and screendetected breast cancers diagnosed in 2005. NHS Breast Screening Programme, 2009

3. National Institute for Health and Clinical Excellence. Guideline 80: Early and locally advanced breast cancer. Diagnosis and treatment. National Institute for Health and Clinical Excellence, 2009.

4. Chung A and Giuliano A. Axillary staging in the neoadjuvant setting. Ann Surgical Oncol, 17, 24012410.

5. Bedi DG, Krishnamurthy R, Krishnamurthy S, et al. Cortical morphologic features of axillary lymph nodes as a predictor of metastasis in breast cancer: in vitro sonographic study. AJR Am J Roentgenol 2008; 191(3): 646-652.

6. Alvarez S, Anorbe E, Alcorta $\mathrm{P}$, et al. Role of sonography in the diagnosis of axillary lymph node metastases in breast cancer: a systematic review. AJR Am J Roentgenol 2006; 186(5): 1342-1348.

7. Abe H, Schmidt RA, Kulkarni K, et al. Axillary lymph nodes suspicious for breast cancer metastasis: sampling with US-guided 14-gauge core-needle biopsy-clinical experience in 100 patients. Radiology 2009; 250 (1): 41-49.

8. Lee MC, Eatrides J, Chau A, et al. Consequences of axillary ultrasound in patients with T2 or greater invasive breast cancers. Ann Surg Oncol. 2010; 18: 727.

9. Fernandez AG, Fraile M, Gimenez N, et al. Use of axillary ultrasound, ultrasound-fine needle aspiration biopsy and magnetic resonance imaging in the preoperative triage of breast cancer patients considered for sentinel node biopsy. Ultrasound Med. Biol. 2011; 37: 16-22.

10. Park SH, Kim MJ, Park BW, et al. Impact of preoperative ultrasonography and fine-needle aspiration of axillary lymph nodes on surgical management of primary breast cancer. Ann Surg Oncol. 2011; 18: 738-44.

11. Baruah BP et al. Axillary node staging by ultrasonography and fine-needle aspiration cytology in patients with breast cancer. Br J Surg 97(5):680-683. 
12. Leenders MWH, Broeders $\mathrm{M}$, Croese $\mathrm{C}$, et al. Ultrasound and fine needle aspiration cytology of axillary lymph nodes in breast cancer. To do or not to do.The Breast 2012; 21: 578-583.

13. Feng Y, Huang R, He Y, Lu A, Fan Z, Fan T, et al. "Efficacy of physical examination, ultrasound, and ultrasound combined with fine-needle aspiration for axilla staging of primary breast cancer" Breast Cancer Research and Treatment. 2015;149(3):761-5.

14. A Moore, M Hester, M-W Nam et al. Distinct Lymph Nodal Sonographic Characteristics In Breast Cancer Patients At High Risk For Axillary Metastases Correlate With Final Axillary Stage. British J Radiol (2008); 81:630-36.

15. Bonnema J, van Geel AN, van Ooijen B, Mali SP, Tjiam SL, Henzen-Logmans SC et al. Ultrasoundguided aspiration biopsy for detection of nonpalpable axillary node metastases in breast cancer patients: new diagnostic method. World J Surg 1997;21(3):270e4.
16. Carroll PA, Mahony DO, McDermott R, et al. Perioperative diagnosis of the positive axilla in breast cancer: A safe, time efficient Algorithm. EJSO 2011; 37: 205-210.

17. Krishnamurthy R, Bedi DG, Krishnamurthy S, Edeiken B, Fornage B, Hunt KK. Ultrasound of axillary lymph nodes: classification based on cortical morphology. Radiology.2001;21(Suppl. P):646.

18. Verbanck J, Vandewiele I, DeWinter HD, Tytgat T, Aelst VF,Tanghe W. Value of axillary ultrasonography and sonographically guided puncture of axillary nodes. A prospective study in 144 consecutive patients. $J$ ClinUltrasound. 1997; 25:53-56.

19. Holwitt DM, Swatske ME, Gillanders WE, Monsees BS, Gao F, Aft RL, et al. The combination of axillary ultrasound and ultrasound-guided biopsy is an accurate predictor of axillary stage in clinically node-negative breast cancer patients, Am J Surg. 2008;196(4):477-82.

\section{Please cite this article in press as:}

V.K Sharma., Vibhor Nanda., K.J.S. Jaswal., Anupam Jhobta and Sudershan Sharma (2017), Role of ultrasound guided fnac in axillary staging in breast carcinoma with no clinically palpable axillary lymph nodes. a prospective study, International Journal of Current Advanced Research, 6(3), pp. 2372-2375. http://dx.doi.org/10.24327/ijcar.2017. 2375.0012 